\title{
Effective Capacity Allocation for Robustness of Interconnected Power Grids
}

\author{
Zhengqi Wu, Zhan Xu, Jingjing Wang*, Sanghai Guan, Xuyang Hou and Yong Ren \\ Department of Electronic Engineering, Tsinghua University, Beijing, 100084, China \\ ${ }^{*}$ Corresponding author
}

\begin{abstract}
In this paper, we study how to allocate the capacity of links in order to both restrain the propagation of cascading failures and to improve the robustness of the whole power grid system. Based on related works, we propose a graph-aided model which maps the real power grid into a graph. It can be viewed as a benchmark of the power grids robustness. Furthermore, a simulated annealing (SA) algorithm is proposed to optimize the allocation of links' capacity. Finally, sufficient analysis is presented and the index $R$ is defined to measure the proposed capacity allocation of interconnected power grids, which is beneficial in terms of configuring the capacity of coupling edges.
\end{abstract}

Keywords-component: interconnected power grid; fixed edge; capacity allocation; robustness

\section{INTRODUCTION}

As an indispensable part of our daily life, the power grid attracts a range of attentions around the world. The power grid may not only be capable of transmitting the energy under normal circumstances, but also of guaranteeing its safe and stable operation under emergencies.

Considering the increasing demand of power systems, power networks are no longer isolated. In [1], Brummitt et al. discovered that the American power grids are constructed by three main parts, i.e. Eastern, Western and Texas. The interconnected edges between these three grid networks were sparse in contrast to their internal edges. Moreover, these interconnected edges played a vital role in the robustness of the whole system [2].

Relying on the definition of 'scale-free' and 'small world' [3] [4], the complex network theory has become a powerful tool to study large-scale networks, such as the Internet, social networks [5], vehicular networks [6], etc. In the state of the art, the complex network theory is conducive to further improve the reliability and efficiency of power grids. Specifically, Zhang et al. [7] proposed a model that abstracted the real power grid into a complex network and studied the reliability of the power grid through the analysis of its topology characteristics. Moreover, the cascading failure of interconnected scale-free network under intentional attack was discussed in [8], where the network can reach the best performance based on the assortative coupling. Sparse interconnected edges were capable of alleviating the cascading failures [9], while the case may get worse when the interconnected edges became dense. It can be concluded that both the property and the quantity of the interconnected edges have an impact on the robustness of the network [10].
Hence, inspired by the above-mentioned issues, in this treatise, we focus our attention on how to allocate the capacity of the interconnected edges in order to restrain the propagation of the cascading failure and improve the robustness of the whole power grid. Our contributions can be summarized as follows. Firstly, we propose a graph aided model to formulate the power grid from both complex and electrical perspectives. Secondly, an optimization algorithm is provided to optimize the capacity allocation, which is robust to certain target attacks. Finally, we propose the revised index $R$ to elaborate the effect of the capacity allocation.

\section{SySTEM MODEL}

\section{A. Basic Model}

In this section, we map the power grid into a graph. We consider power grid with $n$ nodes, and its constraint equations can be given by:

$$
\left[\begin{array}{cccc}
Y_{11} & -Y_{12} & \ldots & -Y_{1 n} \\
-Y_{21} & Y_{22} & \ldots & -Y_{2 n} \\
\vdots & \vdots & \ddots & \vdots \\
-Y_{n 1} & -Y_{n 2} & \ldots & Y_{n n}
\end{array}\right]\left[\begin{array}{c}
v_{1} \\
v_{2} \\
\vdots \\
v_{n}
\end{array}\right]=\left[\begin{array}{c}
I_{1} \\
I_{2} \\
\vdots \\
I_{n}
\end{array}\right] .
$$

where $Y_{i j}(i \neq j)$ is the admittance of the transmission line connecting node $i$ and $j$, and $Y_{i j}=0$, if there is no transmission line. Moreover, $Y_{i i}=\sum_{i \neq j} Y_{i j}$. $v_{i}$ represents the voltage of the node $i$, while $I_{i}$ denotes the external injected current of the node $i$. Each of these equations satisfies Kirchhoff's law,

We divide these nodes into two categories:

1) Consumer Node: Consumer node $i$ consumes energy, and the external injection current $I_{i}$ means the current it consumes. Its constraint equation can be formulated as:

$$
\left[\begin{array}{lllll}
-Y_{i 1} & \cdots & Y_{i i} & \cdots & -Y_{i n}
\end{array}\right] \cdot \mathbf{V}=I_{i}
$$

where $\mathbf{V}=\left[\begin{array}{lllll}\cdots & v_{i} & v_{j} & v_{k} & \cdots\end{array}\right]^{\mathrm{T}}$.

2) Generation Node: Generation node $k$ is a fixed voltage source. The current is generated from the node and depends on its voltage, energy consumption of others and the network topology. Its nodal equation is denoted as:

$$
\left[\begin{array}{lllll}
0 & \cdots & Y_{k} & \cdots & 0
\end{array}\right] \cdot \mathbf{V}=v_{k}
$$


where $Y_{k}=1$ and $v_{k}$ represents the fixed voltage of node $k$.

Combining Eq. (1), Eq. (2) and Eq. (3), we have:

$$
\mathbf{A} \cdot \mathbf{V}=\mathbf{B},
$$

where

$$
\mathbf{A}=\left[\begin{array}{ccccccc}
\ddots & & \cdots & & \cdots & & \cdots \\
Y_{i 1} & \cdots & Y_{i i} & Y_{i k} & \cdots & Y_{i n} \\
0 & \cdots & 0 & y_{k} & \cdots & 0 \\
\cdots & & \cdots & & \cdots & \ddots
\end{array}\right],
$$

as well as

$$
\mathbf{B}=\left[\begin{array}{llll}
\cdots & I_{i} & v_{k} & \cdots
\end{array}\right] .
$$

The nodes subscripted $i$ represent consumer nodes, nodes subscripted $j$ represent generation nodes. Given the energy consumption, the production capacity and the network topology, the voltage values of each node can be calculated by Eq. (4). Hence, the current between node $i$ and node $j$ can be calculated as:

$$
I_{i j}=\left(v_{i}-v_{j}\right) Y_{i j} .
$$

Thus, we abstract the power grid into a network, so that we can analyze the power grid using the complex network theory. Moreover, since the Eq. (4) is derived from the Kirchhoff's law, the production in the network should be identical to the consumption. The output of the power grid depends on the voltage and current of the generation nodes, while the consumption of the power grid depends on the voltage and current of the consumer nodes. If the topology of the network changes, the voltage in the grid will also change. Therefore, the voltage of each node needs to be recalculated in order to ensure the energy conservation of the grid.

\section{B. Cascading Failure}

In the real world, large-scale blackouts are often caused by the collapse of individual nodes. When an element in the network fails, the voltage and current of all nodes in the network will change. The node may fail to work if its load is larger than its capacity. This failure will in turn lead to changes on the voltage and current of other successive nodes. This failure spreads until the load of every surviving node in the network is overladen. This process is named as cascading failure, which can be divided into the following three steps [7]:

1) Initial Setting: Before the cascading failure process begin, we should pre-set the voltage of the generation nodes, the current consumed by the consumer nodes as well as the admittance of transmission line between nodes. In order to weaken the influence of other factors on the experiment, we set the voltage of the generation nodes to 1 p.u.. The injected current at the consumer nodes is set to 20p.u., and the admittance of transmission line is set to 11 p.u.. With these initial settings, the voltage and current of each node and link will be calculated according to Eq. (4). The load $P_{i}$ of the node $i$ is defined as the product of the voltage $v_{i}$ and the external injection current $I_{i}$, i.e., $P_{i}=I_{i} v_{i}$. The capacity of the node is defined as $(1+\alpha)$ times of the initial load. Moreover, the load of the edge is defined as the current that flows across the edge. And the capacity of the edge is defined as $(1+\beta)$ times of the initial load. Here, $\alpha$ and $\beta$ are the safety bounds for nodes and links. Nevertheless, due to economic considerations, the security bound is often limited to a low level.

2) Set Initial Failure: After establishing the initial environment, an element is selected as the first element of the collapse to be removed from the network. If a node is selected as the collapsed element, all connected edges associated with it will be removed from the network.

3) Cascading Iteration: When the first collapsed element is removed from the network, the topology of the network changes. Removal of a node may cause the original connected network to become several subnets that separate from each other. If a subnet has no generation node, it is invalid and needs to be removed together. If a subnet has at least one generation node, the load values of each node and link need to be recalculated according to Eq. (4). The recalculated load value will be compared with the capacity. Specifically, if the load is higher than the capacity, the node or edge is invalid. This process will continue until no element fails. At this point, we achieve the final balanced network.

\section{Robustness Index}

Robustness is the characteristic of a system that maintains some of its performance under certain disturbance. In terms of power systems, robustness is defined as the ability of a system to provide normal service in the event of partial failure. The scale of outage area is used to measure the magnitude of blackouts. According to [7], the importance of the node can be described by the percentage of the outage area caused by the failure node. Here, we define PUN $(i)$ as the percentage of unserved nodes caused by the failure, and we have:

$$
\operatorname{PUN}(i)=\frac{n_{\text {unserved }}(i)}{n} \text {, }
$$

where $n_{\text {unserved }}(i)$ indicates the number of failed nodes caused by the failure of node $i$, while $n$ is the total number of nodes in the network. A large PUN means that the failure of this node may destroy the entire network, whereas a small PUN indicates the failure of this node has little impact on the whole network.

As for power grids, target attacks are more likely to occur on nodes with large load. Therefore, we define the node with the largest initial load as the robustness of the whole network to the target attack, which can be represented as:

$$
\mathrm{PUN}=\mathrm{PUN}(m) \text {, }
$$


where $m$ represents the node with the largest initial load in the network. If the PUN of the network is high, it indicates more failure nodes will be caused by cascading failure under target attack following a poor network robustness. Correspondingly, if the network's PUN value is small, the network has a strong robustness.

\section{OPTIMIZATION AlgORITHM AND SIMULATIONS}

In this section, we use the IEEE bus system for our simulation. According to the model mentioned before, the electrical parameters of the independent grid are calculated according to Eq. (4). The element's capacity is set as $(1+\alpha)$ times of the initial load.

Hence, we build the interconnected network after the parameters of the independent grid are obtained. In our simulation, there are two independent networks, namely Network I and Network II. To eliminate the interference, we assume that a node in Network $I$ is only connected to one node in Network II and vice versa. The connection mode is random connection. The edge connecting Network I and the Network II is called the interconnected edge with its admittance set to 11 p.u.

This paper focuses on the influence of the capacity allocation of the interconnected edge on the robustness of the whole network. The capacity allocation of the interconnected edge is denoted as:

$$
\mathbf{S}=\left[\begin{array}{lllll}
c_{1} & \cdots & c_{i} & \cdots & c_{n}
\end{array}\right],
$$

where $n$ denotes the number of interconnected edges, and $c_{i}$ represents the capacity of edge $i$. Gross $=\sum c_{i}$ is a constant value, and subjects to $0<c_{i}<$ Gross. Recalculating the constructed network according to Eq. (4), we can get a group of initial load for the interconnected edge, i.e.,

$$
\mathbf{I}=\left[\begin{array}{lllll}
I_{1} & \cdots & I_{i} & \cdots & I_{n}
\end{array}\right]
$$

Let Gross $=(1+\alpha) \sum I_{i}$. In order to make the system more robust, we introduce the optimization algorithm to configure $\mathbf{S}$.

The simulated annealing algorithm comes from the principle of solid annealing. When the solid is heated to full height, the particles inside the solid become disordered. Then, it cools slowly and the particles return to an orderly state. Finally, the ground state is returned at normal temperature. At this point, the energy can be reduced to the minimum. In our study, the particle state corresponds to the capacity configuration $\mathbf{S}$, and the energy corresponds to the PUN. First, $\mathbf{S}_{i}$ is randomly generated as the initial state, which can be regarded as an unordered state, and the corresponding energy is very high. Then, we make state $\mathbf{S}_{i}$ jump to another state $\mathbf{S}^{\prime}$. If the energy of the new state $\mathbf{S}^{\prime}$ is lower than the previous state, $\mathbf{S}^{\prime}$ is accepted as a new state. Otherwise, $\mathbf{S}^{\prime}$ is accepted as a new state at a certain probability $r$, which can be calculated as follows:

$$
r=\exp \left(\frac{E\left(\mathbf{S}^{\prime}\right)-E(\mathbf{S})}{k T}\right)
$$

where $E\left(\mathbf{S}^{\prime}\right)$ denotes the energy of $\mathbf{S}^{\prime}$, and $E(\mathbf{S})$ denotes the energy of $\mathbf{S}$. Moreover, $k$ represents the Boltzmann constant, while $T$ is the current temperature. This rule is the Metropolis criterion, which prevents the algorithm from entering the local optimum. The Metropolis criterion makes it possible to accept solutions that differ greatly from the current state at higher temperatures, but only solutions that are less different from the current state can be accepted at low temperatures. When the temperature approaches infinitely low, only solutions with energy lower than the present state can be accepted. This guarantees the system can get to the optimum solution when the temperature is low.

Through the pre-analysis of $\mathbf{S}$, we can conclude that the capacity of the interconnecting edge shall be greater than its initial load value. In other words, $c_{i}$ should meet the following constraints:

$$
I_{i}<c_{i}<\text { Gross }
$$

The specific steps of the optimization algorithm are as follows:

1) Initial setting: The initial temperature is $T=1$, and the initial solution $\mathbf{S}_{i}$ is generated randomly. The objective function $C(\mathbf{S})$ is the PUN of the network. The iteration number is $n$ at each $T$ value. The ending condition is $T<T_{\text {threshold, }}$ or when successive iterations yield the same results.

2) $k$ represents the number of steps to iterate under the current $T$ value. If $k<n$, the 3 and 4 step are performed.

3) Generate the new $\mathbf{S}_{k}^{\prime}$. Increase the $k$-th value in $\mathbf{S}$ by one unit, and ensure that the total capacity is equal to Gross.

4) Calculate the evaluation function $C\left(\mathbf{S}_{k}^{\prime}\right)$ of $\mathbf{S}_{k}^{\prime}$.

5) We obtain $n C\left(\mathbf{S}_{k}^{\prime}\right)$, and mark $\mathbf{S}^{\prime}$ as the set of $c_{i}$ corresponding to the minimum of the $n C\left(\mathbf{S}_{k}^{\prime}\right)$.

6) Calculate increments $\Delta T=C\left(\mathbf{S}^{\prime}\right)-C(\mathbf{S})$. If $\Delta T<0$, accept $\mathbf{S}^{\prime}$ as the new solution, or $\mathbf{S}^{\prime}$ is accepted as the new solution in probability $\exp (-f(\Delta T) / T)$.

7) If the ending condition is satisfied, the current solution is outputted as the optimal solution. Otherwise, $K=0$ and $T$ is reduced. Move to step 2.

In step 6, the Metropolis criterion is adopted to prevent the system from being trapped into the local optimum. In order to improve the efficiency of the algorithm, we introduce the linear processing to $\Delta T$, under the Metropolis criterion. Let $\Delta T$ shrink 10 times, and make it less possible for the system to accept a solution that differs from the current state. This allows the algorithm to converge faster. However, it increases the possibility that the algorithm falls into the local optimal solution. In order to compensate for this defect, the optimization algorithm can be repeated numerous times and we take the optimum solution as the final state.

In the simulation, IEEE30 bus system is used for testing. We can get the results shown in Fig. I(a). The simulation results take the average values from 10 different stochastic networks. As can be seen, the robustness of the capacity allocation obtained by the optimization algorithm is always better than that of the fixed margin capacity allocation. It is worth noting that, with the 
increase of alpha, the total capacity of the interconnected edge will also increase. However, from the data that set capacity $(1+$ $\alpha$ ) times of the interconnected edge's initial load, we can see that PUN is not strictly decreased with the increase of $\alpha$. Let's assume that there is a link $E$ that connects $M$ to $N$, and the voltage of $N$ is higher than $M$ 's. When $\alpha$ gets smaller, $E$ has less capacity and collapses during cascading failure. Therefore, $M$ does not have to sustain the load from $N$. When $\alpha$ gets larger, the capacity of $E$ gets larger as well, and there is no collapse during cascading failure. $M$ will bear the load from $N$, and this part of load may cause $M$ to exceed its capacity and collapse. Here we only change the capacity of the edge, and do not change the capacity of the node. As a result, the behavior of the node is not necessarily related to the capacity of the link. PUN may also appear no decreasing as $\alpha$ increases.
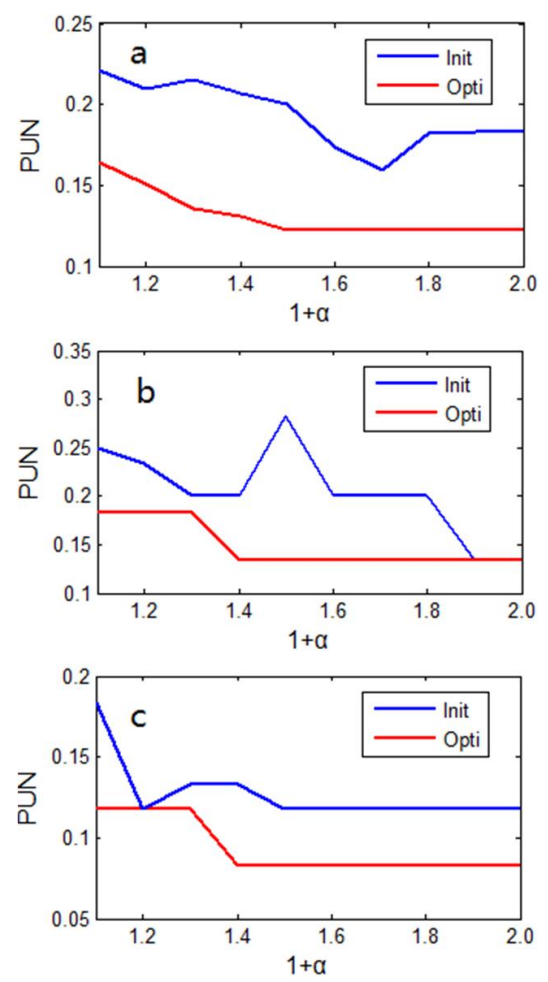

FIGURE I. OPTIMIZATION RESULTS FOR THE IEEE30 BUS SYSTEM. THE BLUE LINE REPRESENTS THE PUN VALUE CORRESPONDING TO S PARAMETERED BY CI $=(1+\mathrm{A}) \mathrm{II}$, AND THE RED LINE INDICATES THE PUN VALUE CORRESPONDING TO THE OPTIMIZED S.

For some topological networks, when $\alpha$ is large, the total capacity is large enough so that it no longer constitutes a major factor affecting PUN. The results obtained by the optimization algorithm are consistent with the performance of the fixed allocation, as shown in Fig. I(b). In some topologies, for the reason mentioned above, even if $\alpha$ is large enough, the same effect cannot be achieved, as shown in Fig. I(c).

However, the PUN getting from the optimization algorithm is always monotonically decreasing. Hence it can be proved that our algorithm gets the optimum result.

\section{RESUlt ANALYSIS}

There are two different approaches for studying the robustness of power grids from the view of complex networks. One is to study the topological characteristics of the network, such as the degree of node, betweenness, clustering coefficient and so on. The other is starting from the electrical property of the network, such as electrical distance, etc..

With the continuous development of power system, power grids are becoming more and more complex. Previous studies pointed out that large-scale power grid often presents a scalefree property, where only a few nodes have large degree and the failure of these nodes is more likely to cause large-scale accidents. Therefore, it is necessary to study the topological characteristics of power grid from this point of view [11].

In our model, the activity of the current is bound by Kirchhoff's law, not just the abstract activity on the network. The network behavior is closely related to the electrical property of the network. Therefore, it is necessary to study the capacity allocation of the interconnected edges from the view of electrodynamics.

The load on the interconnected edge is defined as the magnitude of the current flowing through the edge. Since the admittance of the interconnecting edge is set for 11 p.u., we consider the voltage between the two ends of the interconnected edge. The join of the interconnected edge will change the topological structure of the network, which will also lead to the redistribution of the energy in the whole network. Therefore, only the voltage cannot explain the results precisely. Among the commonly used topological properties, the degree, node betweenness and clustering coefficients are all about the nodes, failing to reflect the property of the edges. Thus, we give priority to the edge betweenness. Edge betweenness measures the importance of interconnected edges in the network.

It is important to note that the topological characteristics of the network are abstract and do not require field measurements and can be obtained only by giving a predefined network structure. Electrical characteristics require field measurements, and capacity needs to be pre-allocated before wiring. Therefore, the voltage data should be the voltage of the independent grid before the interconnection is built.

In this model, generation nodes and consumer nodes are different kinds of node with different behaviors, and need to be considered separately. When the topology of the network changes, the voltage of the generation nodes remains unchanged at 1p.u., but the voltage of the consumer nodes changes accordingly. Thus, when the structure of the network changes, the load on the interconnected edge connected to only one generation node will be greater, and more capacity should be allocated to such an interconnected edge.

Based on the analysis above, we propose the index $R$ to estimate the capacity allocation of the interconnected edges, i.e.,

$$
R_{i}=V_{i} \cdot B c_{i}+G_{i} \cdot C
$$

where $V_{i}$ represents the voltage between the two ends of the edge $i$, and $B c_{i}$ represents the betweenness of the edge $i$. The $G_{i}$ 
indicates whether the two nodes on the interconnected edge contain generation node, which can be calculated as:

$$
G_{i}= \begin{cases}1, & \text { if one of the nodes is a generation node, } \\ 0, & \text { otherwise. }\end{cases}
$$

If both of the nodes are generation nodes, there is no current flowing on the edge, and $G_{i}=0, R_{i}=0$. Moreover, $C$ is a constant which approximately equals to the maximum value of $V \cdot B c$.

Fig. II(a) illustrates the simulation results obtained by the IEEE30 bus system. The network is made up of two IEEE30 bus system and the number of interconnecting edges is 30 . The 30 points in the graph represent 30 interconnecting edges. The abscissa of each point represents the capacity of this edge obtained by the optimization algorithm, and the ordinate is the $R$ corresponding to this edge. To better illustrate the role of $R$, we linearly adjust $R$ to the same order of magnitude as $\mathbf{S}$. As we can see from the figure, the $R$ has a strong positive correlation with the results of the optimization, that is to say, if the $R$ value of the interconnected edge $i$ is smaller, its corresponding capacity is smaller, and vice versa
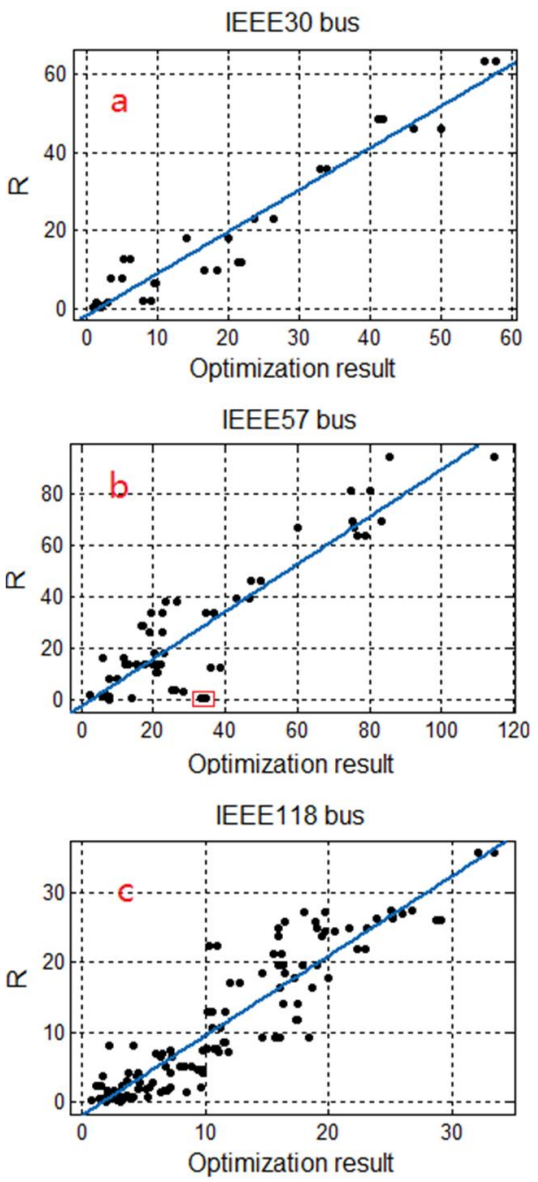

FIGURE II. THE INDEX R VERSUS THE OPTIMIZATION RESULTS.
Fig. II(b) shows the results obtained by the IEEE57 bus system, with the number of interconnected edges being 57 . There are several differences between the 57 bus system and the 30 bus system. Specifically, the distribution of generation nodes in 57 bus system is very centralized, so that some nodes are directly connected to more than two generation nodes. The voltage of these nodes can also be considered essentially unchanged. When calculating the $R$, these nodes can be counted as 0.5 generation nodes. As can be seen from the figure, the vast majority of points still obey a positive correlation. Only the points circled by the red box are far from the fitting curve. The capacity obtained from the optimized configuration is much higher than the calculated $R$. Analysis of the two data shows that the voltage on these two edges is extremely small, approximately 0.3088 , and the mean value foe voltage is 27.1143 . However, the betweenness of the two edges is very high, about 238.3917, which is far from the mean value 122.8574. Moreover, since the voltage is too small, multiplying $V$ with $B c$ does not make up for it. But these two edges are very important in the network, and its capacity should be greater. This situation is very special, so there is no need to adjust the calculation method of $R$ for it. It also shows the topological property of network plays an important role in explaining the behavior of power grid.

Fig. II(c) provides the results of the IEEE118 node system, and we can see that the positive correlation between the $R$ value and the optimized capacity allocation is very strong.

\section{CONCLUSION}

In this paper, we study the effect of capacity of interconnected edges on robustness of interconnected power grids. It is found that both the topological and electrical characteristics of the interconnected edges affect the capacity allocation. The betweenness of the edges indicates the centrality of the edge in the network, and the voltage affects the current of the edges. Taking into account the different types of nodes in the network, we propose the parameter $R$ to measure the size of the capacity of the interconnected edges. The simulation results show that the size of $R$ has a strong positive correlation with the optimum capacity allocation, which can further guide the allocation of the capacity.

\section{REFERENCES}

[1] C. D. Brummitt, R. M. D'Souza, and E. A. Leicht, "Suppressing cascades of load in interdependent networks," Proceedings of the National Academy of Sciences of the United States of America, vol. 109, no. 12, pp. E680-E689, 2012.

[2] L. Cuadra, S. Salcedo-Sanz, J. D. Ser, S. Jimnez-Fernndez, and W. G. Zong, "A critical review of robustness in power grids using complex networks concepts," Energies, vol. 8, no. 9, pp. 9211-9265, 2015.

[3] D. J. Watts and S. H. Strogatz, "Collective dynamics of 'small-world' networks," Nature, vol. 393, no. 6684, pp. 440-442, 1998.

[4] A. L. Barabasi and R. Albert, "Emergence of scaling in random networks," Science, vol. 286, no. 5439, pp. 509-512, 1999.

[5] J. Wang, C. Jiang, T. Q. Quek, X. Wang, and Y. Ren, "The value strength aided information diffusion in socially-aware mobile networks," IEEE Access, vol. 4, pp. 3907-3919, 2016.

[6] J. Wang, C. Jiang, L. Gao, S. Yu, Z. Han, and Y. Ren, "Complex network theoretical analysis on information dissemination over vehicular networks,", 2016 IEEE International Conference on Communications (ICC), 2016, pp. 1-6. 
[7] X. Zhang and K. T. Chi, "Assessment of robustness of power systems from a network perspective," IEEE Journal on Emerging \& Selected Topics in Circuits \& Systems, vol. 5, no. 3, pp. 456-464, 2015.

[8] F. Tan, Y. Xia, W. Zhang, and X. Jin, "Cascading failures of loads in interconnected networks under intentional attack," Europhysics Letters, vol. 102, no. 2, p. 28009, 2013.

[9] S. V. Buldyrev, R. Parshani, G. Paul, H. E. Stanley, and S. Havlin, "Catastrophic cascade of failures in interdependent networks." Nature, vol. 464, no. 7291, pp. 1025-1028, 2010.

[10] W. Zhang, Y. Xia, B. Ouyang, and L. Jiang, "Effect of network size on robustness of interconnected networks under targeted attack," Physica A Statistical Mechanics \& Its Applications, vol. 435, pp. 80-88, 2015.

[11] G. A. Pagani and M. Aiello, "The power gridas a complex network: A survey,” Physica A: Statistical Mechanics \& Its Applications, vol. 392, no. 11, pp. 2688-2700, 2013 